\title{
Effect of Mineral Systems Injected with Zinc Sulfide on Arsenite Removal from Aqueous Solution: Part II
}

\author{
Davidson Egirani", Napoleon Wessey, Adedotun Aderogba \\ Faculty of Science, Niger Delta University, Wilberforce Island, Nigeria
}

Email address:

eenonidavidson@yahoo.com (D. Egirani)

\section{To cite this article:}

Davidson Egirani, Napoleon Wessey, Adedotun Aderogba. Effect of Mineral Systems Injected with Zinc Sulfide on Arsenite Removal from Aqueous Solution: Part II. American Journal of Applied Chemistry. Vol. 3, No. 6, 2015, pp. 201-206. doi: 10.11648/j.ajac.20150306.14

\begin{abstract}
Mineral systems of kaolinite, montmorillonite, goethite and their mixtures were investigated to determine their effect on arsenite removal. Experimental studies include characterization and batch mode experiments. This study was in relation to solution composition and ageing relevant to streams and groundwater impacted by arsenic. Sorption isotherms indicated that sorption capacities of the different clay minerals, goethite and their mixtures were dependent on particle size, $\mathrm{pH}$, particle concentration, arsenic concentration and residence time. Batch mode studies at room temperature revealed increase in sorption as $\mathrm{pH}$ was increased. All mineral systems exhibited increase in sorption as initial arsenic concentration increased. All mineral systems exhibited both promotive and non-promotive $\mathrm{Cp}$ effects. The complex behavior of mineral systems over the range of residence time investigated may be attributed to increased hydroxylation of the mineral surface and availability of thiol $(\equiv \mathrm{S}-\mathrm{H})$ and hydroxyl $(\equiv \mathrm{Me}-\mathrm{OH})$ functional groups and reactive sites.
\end{abstract}

Keywords: Particle Particle Size, Sulfidic-Anoxic, Composition, Ageing, Mixed Mineral Systems

\section{Introduction}

The environmental concern on arsenic is related to its anomalous concentration in surface and ground waters and its availability to living beings [1]. Arsenic pollutants discharged by anthropogenic and natural could result in degraded surface and ground water chemistry [2]. Immobilization of arsenic in the environment occurs through precipitation of low-solubility salts and adsorption on soils and sediments [3]. Remediation processes will follow the same principles, and the most common techniques are based on precipitation and adsorption phenomena [4]. The removal of arsenic from contaminated water is controlled by the solution composition namely, $\mathrm{pH}$ and solid concentration, besides the residence time (ageing) of the solid phase in the water. $[5,6]$.

Adsorption may decrease as particle concentration increases (outer sphere complexation) or not be significantly affected as particle concentration increases (inner sphere complexation. ${ }^{22-25}$. Increase in adsorption as particle concentration increases defines promotive particle concentration effect. [7,8]. The solid concentration effect is an anomalous adsorption phenomenon (i.e., the adsorption isotherm declines as particle concentration increases).
Residence time of solid mineral phase in the absence of a sorbate could results in much mineral surface reorganization $[9,10]$.

Studies have confirmed thiol $(\equiv \mathrm{S}-\mathrm{H})$ and hydroxyl ( $\equiv$ Me$\mathrm{OH})$ functional groups on surface of metal sulfides. These amphoteric reactive units could undergo independent protonation and deprotonation reactions to produce reactive sites for sorption $[11,12]$.

The aim of this study is to explore the effect of particle size of single and mixed mineral systems of kaolinitemontmorillonite, kaolinite-goethite and montmorillonitegoethite injected with zinc sulfide on sorption behavior of arsenite in relation to solution composition and ageing. Different sorption characteristics such as $\mathrm{pH}$, solid concentration and residence time (ageing) under sulfidicanoxic condition have been investigated.

\subsection{Theoretical Models and Isotherms}

This study designed an isotherm model derived from Freundlich isotherm model is designed to explain the predicted behavior of mineral systems. [13, 14].

Distribution coefficient used in calculating arsenic sorbed was derived from the Freundlich model Equation (1) [15]. 


$$
S=K d C^{N}
$$

where $\mathrm{S}$ is the sorbed concentration $(\mu \mathrm{g} / \mathrm{kg}), \mathrm{Kd}$ is the distribution coefficient, $\mathrm{C}$ is the equilibrium concentration $(\mu \mathrm{g} / \mathrm{g}$ ), and $\mathrm{N}=1$ is a chemical-specific coefficient derived from the slope of the plot. The empirical model as provided previously to address the mineral-arsenic interactions is given in Equation (2): [15].

Arsenic sorbed difference $=$ arsenic sorbed-arsenic sorbed $_{\text {total }}$

$$
\text { Arsenic sorbedtotal }=\frac{\left(S_{1}+S_{2}+S_{n}\right)}{n}
$$

where arsenic sorbed ${ }_{\text {total }}$ is the theoretical sorption for a $1: 1$ mixed mineral system, $\mathrm{S}_{1}$ is the arsenic sorbed on first single mineral system, and $S_{2}$ is the arsenic sorbed on second single mineral system, $S_{n}$ is the arsenic sorbed on $n$ number of mineral systems and $\mathrm{n}$ is the number of mineral systems.

\subsection{Theoretical Limitations}

Differences between the actual and predicted sorption capacity for the mixed mineral systems is based on the reasoning that: a. there is limited influence of secondary mineral phase development by mineral-arsenic interaction.

b. components of minerals in the mixed mineral system may act as individual networks or chemisorbed species.

c. There is mass differential between the single and mixed mineral systems.

The difference between the actual sorption and the theoretical sorption was used to clarify the effects of mineral mixing on arsenic sorption. Mineral mixing is said to (a) enhances arsenic removal where the difference is positive; (b) depresses arsenic removal where the difference is negative; and (c) have no effect on arsenic removal where no difference exist between arsenic sorbed and theoretical arsenic sorption.

\section{Materials and Methods}

\subsection{System Characterization}

Summary of system characterization is provided (Table 1). All solutions were prepared using deaerated and deionized water. This water was prepared by bubbling purified nitrogen gas through deionized water for at least 24 hours. Deionized water was obtained from a Millipore Milli-Q system (18 M ).

\begin{tabular}{|c|c|c|c|c|c|c|}
\hline \multirow{2}{*}{$\begin{array}{l}\text { Mineral } \\
\text { Kaolinite }\end{array}$} & \multicolumn{3}{|c|}{ Particle size range $(\mu \mathrm{m})$} & \multirow{2}{*}{$\begin{array}{l}\%(<1 \mu \mathrm{m}) \text { colloid } \\
3.00\end{array}$} & \multirow{2}{*}{$\frac{\mathbf{p H} \pm \boldsymbol{\sigma}}{6.05 \pm 0.05}$} & \multirow{2}{*}{$\begin{array}{l}\text { Surface area }(\mathrm{SSA} \pm \boldsymbol{\sigma})(\mathrm{m} 2 / \mathbf{g}) \\
47.01 \pm 0.24\end{array}$} \\
\hline & $15-30$ & $30-45$ & $45-60$ & & & \\
\hline Montmorillonite & $15-30$ & $30-45$ & $45-60$ & 0.53 & $.01 \pm 0.09$ & $10.00 \pm 0.00$ \\
\hline Goethite & $25-50$ & $50-75$ & $75-100$ & 2.92 & $8.05 \pm 0.06$ & $71.05 \pm 0.17$ \\
\hline Kaolinite/mont & $15-30$ & $30-45$ & $45-60$ & 0.97 & $5.01 \pm 0.02$ & $88.05 \pm 0.55$ \\
\hline Mont/goethite & $1-4$ & $4-8$ & $8-12$ & 3.85 & $3.03 \pm 0.04$ & $147.10 \pm 0.50$ \\
\hline Kaolinite/goethite & $25-50$ & $50-75$ & $75-100$ & 0.73 & $3.05 \pm 0.01$ & $79.30 \pm 0.59$ \\
\hline Zinc sulfide & $5-10$ & $10-15$ & $15-20$ & 8.143 & $5.02 \pm 0.03$ & $4.00 \pm 0.00$ \\
\hline
\end{tabular}

Table 1. Characteristics of mineral systems.

Then the water was purged overnight in an anaerobic chamber containing a mixture of $5 \%$ hydrogen and $95 \%$ nitrogen gases.

Clay minerals and zinc sulfide used in this study provided by the Richard Baker Harrison Company and Acros Organics Ltd Goethite was provided by Iconofile Company Inc. These were nitrogen flushed and stored in airtight containers in the anaerobic chamber before use to avoid oxidation. Arsenic(III) stock solution was purchased from Merck. The AAS standard solution of $1000 \mathrm{mg} / \mathrm{L}$ Arsenic(III) was prepared by transferring the contents of a Titrisol ampule with $\mathrm{As}_{2} \mathrm{O}_{3}$ in $\mathrm{H}_{2} \mathrm{O}$ (Merck, Germany) into a volumetric flask, which was filled up to the mark and stored at $20 \pm 2{ }^{\circ} \mathrm{C}$ according to the instructions by Merck. The working solutions of different concentrations were prepared by diluting the stock solution immediately before starting the batch studies [13].

For characterization of the mineral systems, (a) Coulter laser method was used to determine the particle sizes; (b) \% colloid was estimated from the particle size distribution curves; (c) equilibrium $\mathrm{pH}$ of the untreated mineral systems was determined using the Model 3340 Jenway ion meter; (d) the standard volumetric Brunauer, Emmett, and Teller (BET) method was used to determine the surface areas [16, 17], (f) spectral analysis was performed using scanning electron microscopy, energy dispersive spectroscopy and $\mathrm{x}$-ray diffraction to identify the mineral sorbent.

In this study, $1 \%$ acidified zinc sulfide sulfidic-anoxic system was prepared using deoxygenated deionized water. Purified nitrogen gas was bubbled through the zinc sulfide system continuously for 24 hours. The content, securely sealed was stored in airtight containers in the anaerobic chamber in dark environment before use. The formation of hydrogen sulfide was prototypically characterized by a "rotten egg" odor. [18]

Particle size was determined using LS 13320 coulter laser diffraction particle size analyzer. In this study, $1 \%$ mineral system was prepared using drops of sodium hexametaphosphate (calgon solution) and deionized water. The content was placed on ultrasonic bath and stirred for 5 minutes. The content was removed from ultrasonic bath, a magnetic base inserted and content stirred for another 5 minutes. Sample was pipetted into the analyzer and run at $8 \%$.

\subsection{Sorption Experiments}

Batch mode experimental studies were conducted by adding $1 \%$ sulfidic-anoxic system of zinc sulfide was added 
to $1 \%$ single mineral systems of kaolinite, montmorillonite and goethite. Also, 1\% sulfidic-anoxic mineral system of zinc sulfide was added to $1: 1$ mixed mineral system of kaolinite/montmorillonite, kaolinite/goethite and montmorillonite/goethite, used to elucidate the difference in sorption between the single and mixed mineral phases.

For batch mode $\mathrm{pH}$ investigation, 1\% sulfidic-anoxic system of zinc sulfide was added to $1 \%$ single and 1:1 mixed mineral systems made up to $50 \mathrm{ml}$ containing $1 \%$ (by mass) mineral system, reacted with solution containing $15 \mathrm{ppm}$ of arsenite at zero electrolyte background. Treated system was adjusted to the required $\mathrm{pH}$ (ranging from $\mathrm{pH} 4$ to 8 ) using $0.1 \mathrm{M} \mathrm{HNO}_{3}$ and $0.1 \mathrm{M} \mathrm{NaOH}$. The treated systems were equilibrated for 24 hours and $\mathrm{pH}$ measured using a Model 3340 Jenway ion meter.

For batch mode initial metal concentration investigation, 1\% sulfidic-anoxic system of zinc sulfide was added to $1 \%$ single and 1:1 mixed mineral systems made up to $50 \mathrm{ml}$ containing $1 \%$ (by mass) mineral system, reacted with solution containing $10,15,20$ and $40 \mathrm{ppm}$ of arsenite at zero electrolyte background. Treated system was adjusted to the required $\mathrm{pH}$ (ranging from $\mathrm{pH} 4$ to 8 ) using $0.1 \mathrm{M} \mathrm{HNO}_{3}$ and $0.1 \mathrm{M} \mathrm{NaOH}$. The treated systems were equilibrated for 24 hours and $\mathrm{pH}$ measured using a Model 3340 Jenway ion meter.
For batch mode solid or particle concentration investigation, $1 \%$ sulfidic-anoxic system of zinc sulfide was added to $1 \%$ single and 1:1 mixed mineral systems made up to $50 \mathrm{ml}$ containing solid concentrations $(\mathrm{g} / \mathrm{l})$ of $2,4,6,8$ and 10 were reacted with solution containing $15 \mathrm{ppm}$ of arsenite at zero electrolyte background. The treated mineral systems were adjusted to $\mathrm{pH} 4$ and equilibrated for 24 hours.

Batch mode ageing investigations was carried out from 24 to 720 hours. $1 \%$ sulfidic-anoxic system of zinc sulfide was added to $1 \%$ single and $1: 1$ aged mixed mineral systems containing $1 \%$ (by mass were reacted with solution containing $15 \mathrm{ppm}$ of arsenite at zero electrolyte background. The treated systems, adjusted to $\mathrm{pH} 4$ with no added electrolyte, were equilibrated for 24 hours.

In all experimental studies samples were stored in the dark at room temperature $\left(23 \pm 3{ }^{\circ} \mathrm{C}\right)$ for a maximum of 24 hours before analysis. ${ }^{58}$. Supernatant was filtered through a cellulose acetate filter (pore size $0.2 \mu \mathrm{m}$ ) and analyzed for arsenic (III), using a Hitachi Atomic Absorption Spectrophotometer (HG-AAS).

\section{Results and Discussion}

\subsection{Mineral Systems and pH Effect on Arsenite Sorption}

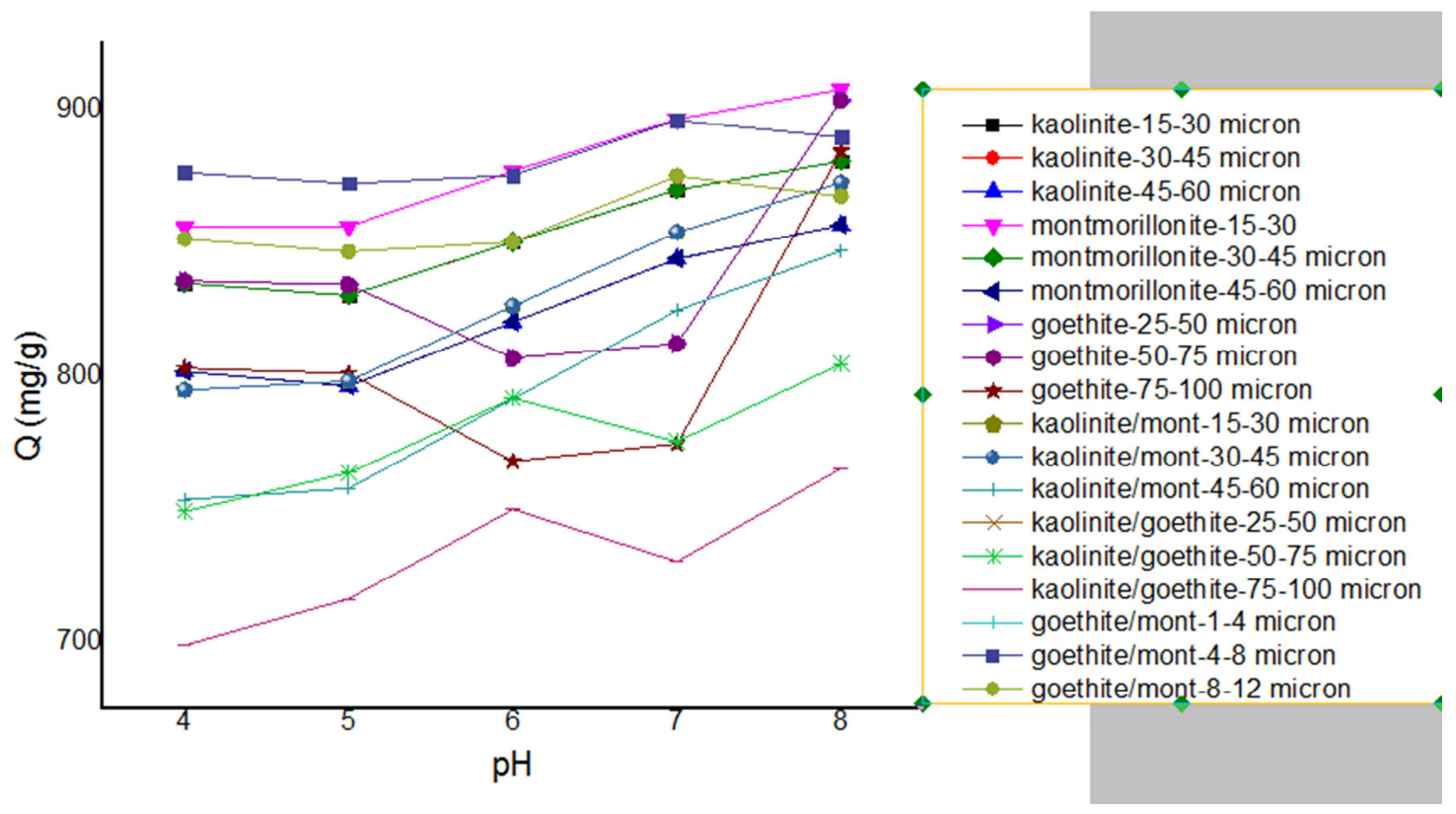

Fig. 1. Plots of arsenic sorbed versus pH on for mineral systems.

In previous study in the absence of sulfidic-anoxic condition [15] arsenic (III) demonstrated a near linear sorption increase with increasing $\mathrm{pH}$ for single mineral systems of kaolinite, montmorillonite, and mixed mineral system of kaolinite/montmorillonite. In this study under sulfidic-anoxic condition and over the range of particle sizes investigated, sorption capacity increases with $\mathrm{pH}$ increase and in a sinusoidal pattern in some cases as shown in Figure 1. $\mathrm{pH}$ effect decreases with increase in particle size for all mineral systems. Kaolinite-goethite exhibit lowest sorption capacity, marginally increasing with $\mathrm{pH}$ increase. Cross over
$\mathrm{pH}$ exist, indicating similar sorption capacity by mineral systems. This complex variability in sorption may be attributed to increased deprotonation of reactive sites as $\mathrm{pH}$ was increased and the effect of thiol $(\equiv \mathrm{S}-\mathrm{H})$ and hydroxyl ( $\equiv \mathrm{Me}-\mathrm{OH}$ ) functional groups and reactive sites in solution.

\subsection{Mineral Systems and Initial Metal Concentration Effect}

In previous study [15], arsenic(III) demonstrated a near linear sorption increase with increase in initial metal concentration for single mineral system of kaolinite, 
montmorillonite, and mixed mineral system of kaolinite/montmorillonite In this study, all mineral systems exhibited linear sorption increase as initial arsenic concentration increased as shown in Figure 2. Sorption decreased with increase in particle size of mineral systems. This variability in sorption may be attributed to availability of thiol $(\equiv \mathrm{S}-\mathrm{H})$ and hydroxyl $(\equiv \mathrm{Me}-\mathrm{OH})$ functional groups on surface of metal sulfides reactive sites.

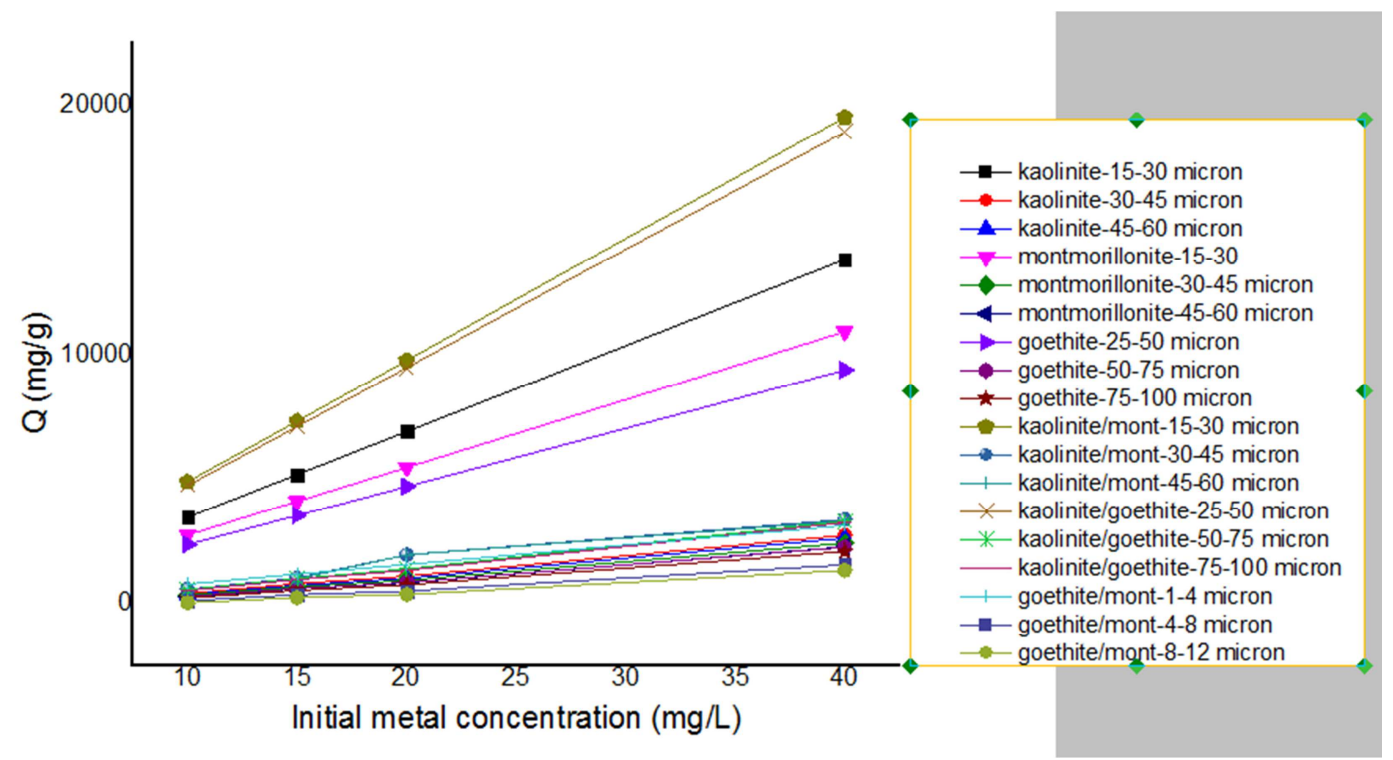

Fig. 2. Plots of sorption capacity versus Initial arsenic concentration for mineral systems.

\subsection{Mineral Systems and Cp Effect}

In previous study in the absence of sulfidic-anoxic condition [15], the behavior of arsenic(III) sorption as particle concentration ( $\mathrm{Cp}$ ) increased was complex being linear for goethite and mixed mineral systems containing goethite.

In this study under sulfidic-anoxic condition, sorption decreased as $\mathrm{Cp}$ increased in some of the mineral systems. In some cases, sorption increased as $\mathrm{Cp}$ increased as shown in Figure 3. Therefore, both promotive and non-promotive $\mathrm{Cp}$ effects exist over the range of particle size and particle concentration investigated. Goethite in mixed systems may form separate (discrete) particles or it may form coatings on other mineral surfaces. Coatings of only a few atomic layers thickness are sufficient to influence sorption rates. This may account for differences in sorption behavior for arsenite sorbed on mixed systems containing goethite.

Decrease in arsenite sorption as $\mathrm{Cp}$ increases may be attributed to increase in particle size and aggregation of the mineral systems. The $\mathrm{Cp}$ effect is also related to effective surface area, pressure, and force at the mineral/water interface.

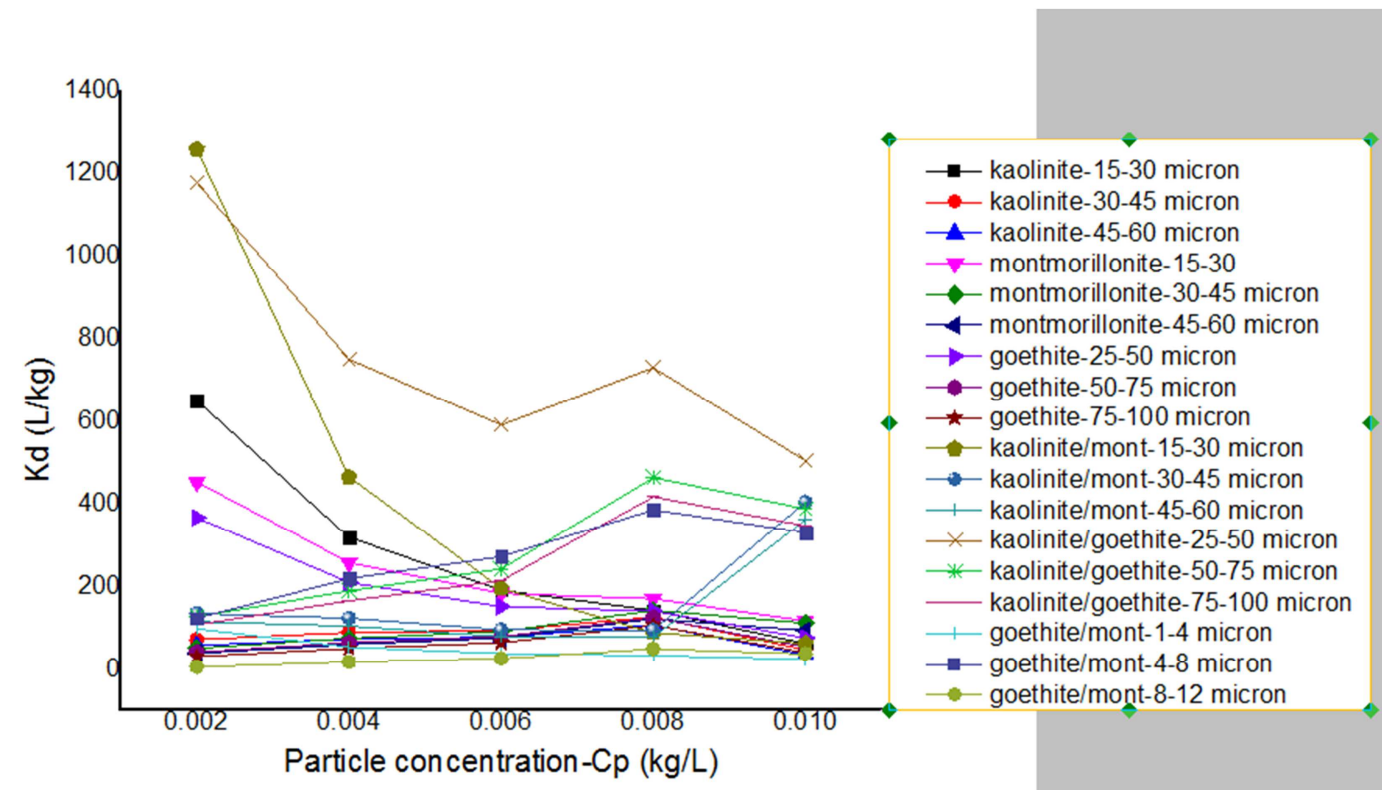

Fig. 3. Plots of actual Kd versus particle concentration for arsenic sorbed on mineral systems. 


\subsection{Mineral Systems and Ageing Effect}

In previous investigation [15] in the absence of sulfidicanoxic condition, all single and mixed mineral systems except kaolinite demonstrated step-wise arsenite sorption. In this study, under sulfidic anoxic condition, Kaolinite over the 15-30 micron range exhibited increase in sorption as ageing was increased. However, all other mineral systems exhibited minimal change in sorption as ageing was increased. The complex behavior of these mineral systems during ageing under sulfidic-anoxic condition may be attributed to the presence of thiol $(\equiv \mathrm{S}-\mathrm{H})$ and hydroxyl $(\equiv \mathrm{Me}-\mathrm{OH})$ functional groups on surface of metal sulfides reactive sites.
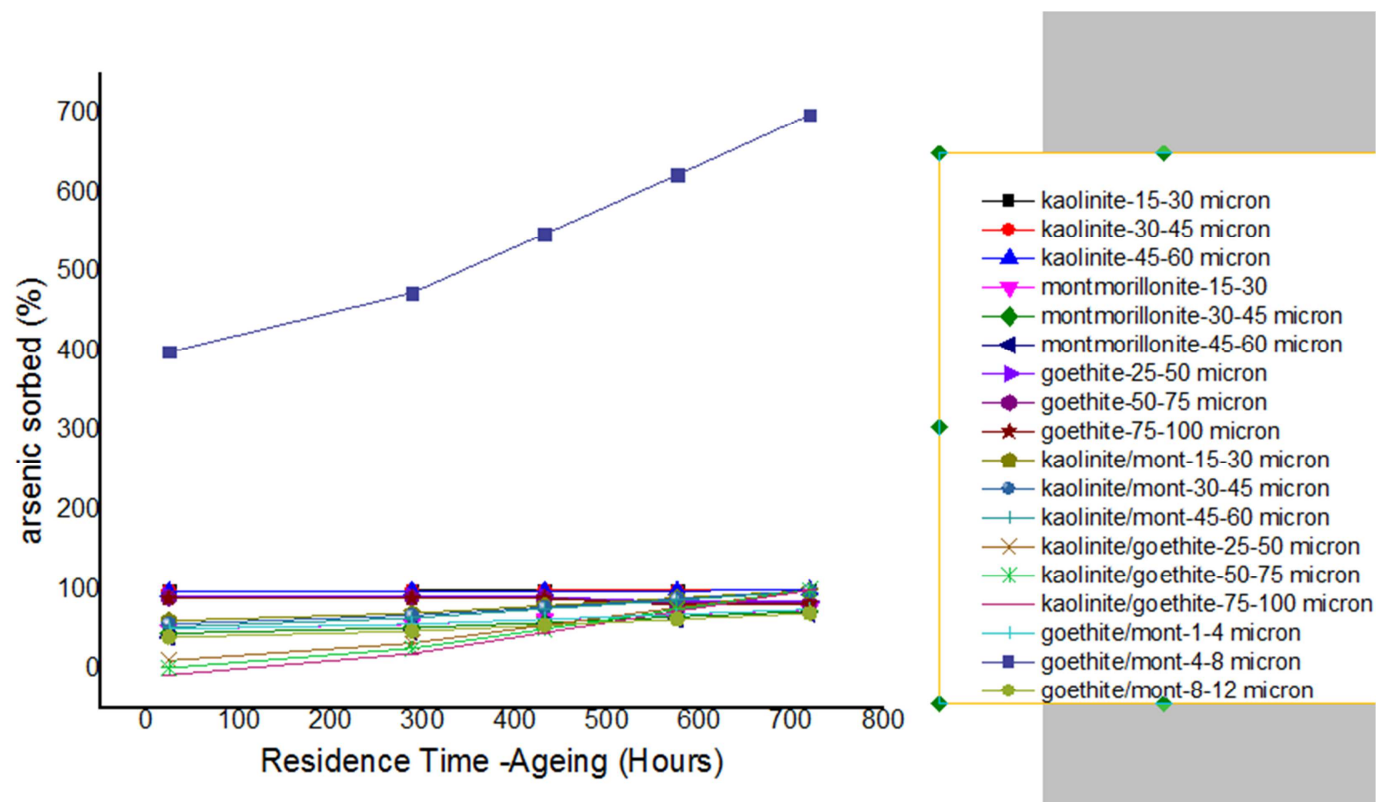

Fig. 4. Plots of arsenic sorbed versus residence time for mineral systems.

\section{Conclusions}

Mineral systems were investigated to determine their effect on arsenite removal. This study was in relation to solution composition and ageing relevant to streams and groundwater impacted by arsenic. Sorption isotherms indicated that sorption capacities of the different clay minerals, goethite and their mixtures were dependent on the particle size, $\mathrm{pH}$, initial metal concentration, particle concentration and residence time. Variability in sorption by these mineral systems may be attributed to increased deprotonation of reactive sites as $\mathrm{pH}$ was increased and the presence of thiol $(\equiv \mathrm{S}-\mathrm{H})$ and hydroxyl $(\equiv \mathrm{Me}-\mathrm{OH})$ functional groups on surface of metal sulfides reactive sites. Both promotive and non-promotive $\mathrm{Cp}$ effects exist over the range of particle size and particle concentration investigated. Sorption pattern appeared to be controlled by outer sphere complexation, inner sphere complexation and intra-particle diffusion. While increase in arsenite sorption with increase in $\mathrm{Cp}$ may be attributed to increase in specific surface area, a decrease in arsenite sorption of the range of $\mathrm{Cp}$ investigated may be attributed to increase in particle size, flocculation and aggregation of mineral system.

All mineral systems exhibited sorption increase as initial arsenic concentration increased. This variability in sorption may be attributed to availability of thiol $(\equiv \mathrm{S}-\mathrm{H})$ and hydroxyl $(\equiv \mathrm{Me}-\mathrm{OH})$ functional groups on surface of metal sulfides reactive sites. All but kaolinite mineral system over the 15-30 micron range exhibited minimal change in sorption as ageing was increased. The variable and complex behavior of mineral systems over the range of residence time investigated may be attributed to increased hydroxylation of the mineral surface resulting in the formation of new reactive sites.

\section{Acknowledgements}

The authors are grateful to the management of Niger Delta University for the release of academic staff allowances. These monies were used for this research work.

\section{References}

[1] Clara, M. Magalhães, F. (2002). Arsenic: An environmental problem limited by Solubility, Pure Appl. Chem., 74(10), $1843-1850$.

[2] Williams. M. (2001). Arsenic in mine waters: an international study. Environ. Geol. 40, 267-278.

[3] Altun, T., \& Pehlivan, E. (2012).Removal of $\mathrm{Cr}(\mathrm{VI})$ from aqueous solutions by modified walnut shells. Food Chemistry, $132,693-700$.

[4] Dupont, L., Jolly, G., \& Aplincourt, M. (2007). Arsenic adsorption on lignocellulosic substrate loaded with ferric ion. Environmental Chemistry Letters, 5, 125-129. 
[5] Chowdhury, S.R., Yanful, E.K., (2010). Arsenic and chromium removal by mixed magnetiteemaghemite nanoparticles and the effect of phosphate on removal. J. Environ. Management, 91 (11), 2238-2247.

[6] Schlegel M. Manceau, A., Charlet, L, Chateigner, D., Hazemann, J.-L., (2001). Sorption of metal ions on clay minerals. III. Nucleation and epitaxial growth of $\mathrm{Zn}$ on the edges of hectoliter. Geochimica et Cosmochimica Acta, 65, 4155-4170.

[7] Davis, J.A and Kent, D.B. (1990). Surface Complexation Modeling in aqueous geochemistry, Review in Mineralogy, 23, 177-260.

[8] Lutzenkirchen, J Ionic Strength Effects on Cation Sorption to Oxides: Macroscopic Observations and Their Significance in Microscopic Interpretation J. Colloid Interface Sci. 2001, 65 149-155.

[9] Matis, K. A, Lehmann, M., and Zouboulis, A. I Modeling sorption of metals from aqueous solution onto mineral particles: The case of arsenic ions and goethite ore. In P. Misaelides, F. Macašek, T. J. Pinnavaia, \& C. Colella (Eds.), Natural microporous materials in environmental technology The Netherlands: Kluwer, 463-472pp. 1999.

[10] Philips, I.R. Copper, lead, cadmium and zinc sorption by waterlogged and air-dry soil J. Soil Contam. 1999, 8 343-364.

[11] Manning, B. A and Goldberg. S. (1997). Adsorption and stability of arsenic(III) at the clay mineral water interface. Environ. Sci. Technol. 31, 2005-2011.
[12] Lin, T. F. and Wu. J. K. (2001). Adsorption of arsenite and arsenate within activated alumina grains: equilibrium and kinetics. Water Res. 35, 2049-2057.

[13] Tournassat, C. Charlet, L. Bosbach, D. Manceau. A. (2002). Arsenic (III) oxidation by birnessite and precipitation of manganese (II) arsenate. Environ. Sci. Technol. 36, 493-500.

[14] Awual, M. REl-Safty S. A. Jyo A Removal of trace arsenic(V) and phosphate from water by a highly selective ligand exchange adsorbent Spectrochimica Acta Part A, 2013, 100 161-165.

[15] Egirani, D.E., Baker, A.R and Andrews, J.E, 2 Arsenite Removal from Aqueous Solution by Mixed Mineral Systems Ii. The Role of Solution Composition And Ageing, International Journal of Recent Scientific Research, 2013, 4 (4), $439-443$.

[16] Brunauer, S. Emmett, P.H. Teller, E. (1938). Adsorption of gases in multimolecular layers, J. Am. Chem. Soc., 60, 309319 .

[17] Zhang, M. Gao B. Varnoosfaderani S. Hebard, A. Yao, Y., Inyang M. (2011). Preparation and characterization of a novel magnetic biochar for arsenic removal, Journal of environmental Sciences, 23(12) 1947-1954.

[18] Wilkin, R.T. and Barnes, H.L. (1996). Pyrite formation by reactions of iron monosulfides with dissolved inorganic and organic sulfur species, Geochimica et Cosmochimica Acta, 60(21), 4167-4179. 